\title{
Opacity distribution in static and moving media
}

\author{
B. Baschek ${ }^{1,3}$, W. v. Waldenfels ${ }^{2,3}$, and R. Wehrse $\mathrm{H}^{1,3}$ \\ 1 Institut für Theoretische Astrophysik, Tiergartenstrasse 15, 69121 Heidelberg, Germany \\ 2 Institut für Angewandte Mathematik, Im Neuenheimer Feld 294, 69120 Heidelberg, Germany \\ 3 Interdisziplinäres Zentrum für Wissenschaftliches Rechnen, Im Neuenheimer Feld 368, 69120 Heidelberg, \\ Germany
}

Received 11 January 2001 / Accepted 21 March 2001

\begin{abstract}
The use of the conventional opacity distribution function (ODF) to deal with very many spectral lines is restricted to static media. In this paper, its generalization to differentially moving media is derived from the analytical solution of the comoving-frame radiative transfer equation. This generalized ODF depends on only two parameters, on the wavelength position (as in the static case) and in addition on a wavelength interval $\Delta$ over which the line extinction is averaged. We present two methods for the calculation of the generalized ODF: (i) in analogy to the static case, it is derived from the mean values of the extinction coefficients over wavelength intervals $\Delta$, (ii) it is calculated under the assumption that the lines follow a Poisson point process. Both approaches comprise the conventional static case as the limit of vanishing velocities, i.e. of $\Delta \rightarrow 0$. The averages of the extinction for all values of $\Delta$ contain the necessary information about the Doppler shifts and about the correlations between the extinction at different wavelengths. The flexible statistical approximation of the lines by a Poisson point process as an alternative to calculating the averages over all $\Delta$ from a deterministic "real" spectral line list, has the advantage that the number of parameters is reduced, that analytical expressions allow a better insight into the effects of the lines on the radiative transfer, and that the ODFs and their corresponding characteristic functions can be calculated efficiently by (fast) Fourier transforms. Numerical examples demonstrate the effects of the relevant parameters on the opacity distribution functions, in particular that with increasing line density and increasing $\Delta$ the ODF becomes narrower and its maximum is shifted to larger extinction values.
\end{abstract}

Key words. radiative transfer - spectral lines - opacity distribution function - stellar atmospheres radiation hydrodynamics

\section{Introduction}

Accurate modeling of spectra of astronomical objects, in particular of stellar atmospheres, usually requires the inclusion of the contribution of a very large number of spectral lines to the opacity. In static media, e.g. in static stellar atmospheres, there are basically three methods which are successfully being applied to deal with the problem of many lines: (i) the direct deterministic calculation of the intensity, (ii) the sampling technique, and (iii) the calculation by means of opacity distribution functions (ODF). In (differentially) moving media, however, only the first method gives correct results, but it is strongly hampered in use by the enormous requirements concerning computer time. The use of the conventional ODF and of the sampling techiques is restricted to static media since the Doppler shift of the spectral lines due to the motions forces the inclusion of an additional correlation of the line extinction with wavelength.

Send offprint requests to: R. Wehrse,

e-mail: wehrse@ita.uni-heidelberg.de
In this paper we present a generalization of the ODF where this correlation is taken care of by an additional parameter $\Delta$, a (logarithmic) wavelength interval over which the line extinction coefficient is averaged. We describe two procedures to calculate the generalized ODF: the first one is based on the direct averaging of the line extinction coefficient from the data contained in a "real" deterministic list over all values of $\Delta$ in a way similar to that of obtaining the conventional static ODF, the other one is a statistical method based on the approximation of the line distribution by a suitable statistical distribution. As has been shown by Wehrse et al. (1998), for this the Poisson point process is well suited and very flexible and can be described by relatively few parameters. The aim of this paper is to provide the basic formulae and a few selected examples, but it is not its purpose to investigate e.g. the influence of the line properties and densities on the generalized ODF although the presentation of the line statistics by a Poisson point process allows considerable insight. 
In Sect. 2 we first review the conventional static opacity distribution function, emphasizing its interpretation as a probability distribution. In order to derive the generalization of the ODF to moving media, it suffices to consider a plane-parallel stratification and to formulate the radiative transfer equation and its well known solution for the specific intensity in terms of expectation values of the radiative quantities. Subsequently it is shown in Sect. 3 that the comoving-frame transfer equation and its analytical solution by Baschek et al. (1997a) directly leads to a "natural" generalization of the opacity distribution function to differentially moving media. Numerical examples for opacity distribution functions from a simulated deterministic line list, for the static as well as for the moving case, are given in Sect. 4. We then turn to the statistic description of the line extinction by means of a Poisson point process: after a short overview over the main results of this statistic description in Sect. 5, we derive in Sect. 6 the generalized ODF expressed in terms of the parameters of the Poisson distributed lines, i.e. mainly in terms of the line density. Section 7 contains numerical calculations of opacity distribution functions for selected parameters from a set of Poisson distributed lines. Finally, we discuss and summarize our results in Sect. 8.

\section{Static opacity distribution function}

A forerunner of the concept of opacity distribution functions is the "fat line" introduced by Labs (1951): the spectrum is subdivided into relatively small wavelength intervals in each of which the line extinction coefficients are shifted together in order to form a single strong line.

The opacity distribution functions, obtained from deterministic line lists by a suitable grouping of the line extinction over small wavelength intervals (Strom \& Kurucz 1966; Mihalas 1978), are - in particular in modeling stellar atmospheres - widely being used to reduce the effort of including a large number of lines in the opacity.

The conventional concept of the opacity distribution function requires that a wavelength interval is considered which on the one hand is narrow enough so that e.g. the continuous extinction and the Planck function do not vary much over it, and within which the exact line positions are of no interest. On the other hand the interval should be large enough to allow a reasonable line statistics. In addition the distribution function is assumed to be independent of e.g. the depth in a stellar atmosphere.

We write the monochromatic extinction coefficient in the form

$\chi(\xi)=\chi_{\mathrm{c}}+\sum_{l=1}^{L} \chi_{l}(\xi)=\chi_{\mathrm{c}}+X(\xi)$

where $\chi_{\mathrm{c}}$ is the continuous extinction coefficient and $X(\xi)=\sum_{l} \chi_{l}(\xi)$ is the contribution of a large number $L$ of spectral lines (each one denoted by $l$ ). In this paper, we use logarithmic wavelengths $\xi=\ln \lambda$. Since in our discussion of opacity distribution functions we are only interested in the line contribution which is a rapidly varying function of the (logarithmic) wavelength $\xi$, we may for simplicity assume that $\chi_{\mathrm{c}}$ is independent of $\xi$.

The line extinction coefficient is usually expressed as

$\chi_{l}(\xi)=A_{l} \cdot \Phi_{l}\left(\xi, \gamma_{l}\right)$,

where $\Phi_{l}$ is the profile function with the intrinsic width $\gamma_{l}$. Its integral over the whole spectral line is normalized by $\int_{\text {line }} \Phi_{l}\left(\xi, \gamma_{l}\right) \mathrm{d} \xi=1$. The integral of $\chi_{l}(\xi)$ over the line is the line strength $A_{l}$.

The classical procedure of calculating the opacity distribution function then assumes that every value $X(\xi)$ is adopted within a certain interval of $\xi$ (ergodic hypothesis), and that the ODF can be determined from the statistics of $X(\xi)$.

A more productive way, however, is to regard the opacity distribution function as the probability $\mathbb{P}$ that the contribution of all $L$ spectral lines to the monochromatic extinction coefficient $X(\xi)$ at any (logarithmic) wavelength $\xi$ has a value between $x$ and $x+\mathrm{d} x$. We then may write this probability as

$\mathbb{P}(X(\xi) \in(x, x+\mathrm{d} x))=p(x ; \xi) \mathrm{d} x$

with the normalization $\int_{0}^{\infty} p(x ; \xi) \mathrm{d} x=1$.

\subsection{Static radiative transfer equation}

In order to discuss the role of the opacity distribution function it is sufficient to consider the simple case of a plane-parallel layer and apply the radiative transfer equation

$\mu \frac{\partial I}{\partial z}=-\chi \cdot(I-S)$

to solve for the monochromatic specific intensity $I=$ $I(z, \xi, \mu)$ in any fixed direction described by $\mu$, the cosine of the angle between the ray and the normal of the layer as which we choose the vertical coordinate $z$. The source function $S=S(z, \xi)$ is assumed to be given. Without affecting the essential effects we want to discuss, we may assume that the extinction coefficient $\chi=\chi(\xi)$ only depends on $\xi$ and not on $z$. As boundary condition we assume that the bottom $(z=0)$ of the layer is irradiated by the intensity $I(0, \xi)$, and that there is no irradiation from above at the top $z$.

Since in plane-parallel geometry the intensity for any direction $\mu$ is trivially obtained from that e.g. for $\mu=+1$, we in addition may restrict ourselves to consider only the outgoing intensity.

\subsection{Solution of the static transfer equation}

The well known solution for the static case (cf. Mihalas 1978) for $\mu=+1$ and for a depth-independent extinction coefficient $\chi(\xi)$ is

$I(z, \xi)=I(0, \xi) \mathrm{e}^{-\chi(\xi) z}+\int_{0}^{z} \mathrm{e}^{-\chi(\xi)(z-\ell)} \chi(\xi) S(\ell, \xi) \mathrm{d} \ell(5)$ 
with $I(0, \xi)$ being the irradiation at the lower boundary $z=0$. The advantages and implications of assuming $\chi(\xi)$ independent of $z$ will be discussed in Sect. 3.1 in the context of the solution of the transfer Eq. (12) for the moving case.

Once the monochromatic intensities are known, the mean intensity as well as the radiative flux can be obtained by integration over $\mu$.

Interpreting the opacity distribution function now as a probability, we have to consider instead of the monochromatic intensity $I$ itself its expectation value $\langle I\rangle$ (in this paper we denote expectation values by acute parentheses $\langle\ldots\rangle)$. In order to obtain $\langle I\rangle$, we have to average the function $\chi(\xi)$ which occurs in the radiative transfer equation and hence in its solution (5):

$$
\begin{aligned}
\langle I(z, \xi)\rangle= & \left\langle\mathcal{I}_{0}\right\rangle+\int_{0}^{z}\left\langle\mathrm{e}^{-\chi(\xi)(z-\ell)} \cdot \chi(\xi)\right\rangle S(\ell, \xi) \mathrm{d} \ell \\
= & \left\langle\mathcal{I}_{0}\right\rangle+S(z, \xi)-S(0, \xi)\left\langle\mathrm{e}^{-\chi(\xi) z}\right\rangle \\
& -\int_{0}^{z}\left\langle\mathrm{e}^{-\chi(\xi)(z-\ell)}\right\rangle \frac{\mathrm{d} S(\ell, \xi)}{\mathrm{d} \ell} \mathrm{d} \ell
\end{aligned}
$$

with the irradiation term

$$
\left\langle\mathcal{I}_{0}\right\rangle=\left\langle I(0, \xi) \mathrm{e}^{-\chi(\xi) z}\right\rangle \text {. }
$$

The second version (Eq. (7)) of the solution has been obtained by partial integration from the first one (before performing the expectation value). It is better suited for studying the opacity distributions since it does not contain the expectation value of the product of $\chi$ with the exponential containing $\chi$.

From the above solution it is seen that the essential expression is the expectation value

$$
\begin{aligned}
\left\langle\mathrm{e}^{-\chi(\xi) \cdot z}\right\rangle & =\mathrm{e}^{-\chi_{\mathrm{c}} \cdot z}\left\langle\mathrm{e}^{-X(\xi) \cdot z}\right\rangle \\
& =\mathrm{e}^{-\chi_{\mathrm{c}} \cdot z} \int_{0}^{\infty} \mathrm{e}^{-x \cdot z} p(x ; \xi) \mathrm{d} x
\end{aligned}
$$

\subsection{Opacity distribution function and the corresponding expectation value}

The expectation value of $\exp (-X z)$ is the Laplace transform $\mathcal{L}$ of the probability $p(x ; \xi)$, i.e. of the conventional opacity distribution function:

$\mathcal{L}_{z}\{p\} \equiv \mathbb{L}(z ; \xi)=\int_{0}^{\infty} \mathrm{e}^{-x \cdot z} p(x ; \xi) \mathrm{d} x=\left\langle\mathrm{e}^{-X \cdot z}\right\rangle$,

where $\xi$ is only a parameter. The inverse Laplace transform of (10) for $x>0$ is

$$
p(x ; \xi)=\mathcal{L}^{-1}\{\mathbb{L}(z ; \xi)\}=\frac{1}{2 \pi i} \int_{\gamma-i \infty}^{\gamma+i \infty} \mathrm{e}^{x \cdot z} \mathbb{L}(z ; \xi) \mathrm{d} z
$$

where $\gamma$ is a real constant that exceeds the real part of all the singularities of $\mathbb{L}(z ; \xi)$ (Gradshteyn \& Ryzhik 1980). For practical purposes, however, Eq. (11) can most conveniently be evaluated by means of a fast Fourier transform.

\section{Opacity distribution function for moving media}

We now turn to the generalization of the opacity distribution function to moving media. In order to discuss the essential features it is sufficient - similarly as for the static case - to consider a plane-parallel layer and apply a simplified version of the comoving-frame radiative transfer equation.

\subsection{Comoving-frame transfer equation}

Assuming small velocities $\beta=v / c \ll 1$, and furthermore neglecting aberration and advection, Wehrse et al. (2000, Eq. (32)) derived the transfer equation for 3D media in the ray direction $z$ (or in the direction of the unit vector $\boldsymbol{n}$ ),

$\frac{\mathrm{d} I}{\mathrm{~d} z}+w \frac{\partial I}{\partial \xi}=-\chi(I-S) \quad$ with $\quad w \simeq \boldsymbol{n} \cdot \nabla(\boldsymbol{\beta} \cdot \boldsymbol{n})$,

of which the plane-parallel transfer equation given e.g. by Mihalas (1978, Eqs. (14-99)) is a special case.

In order to solve Eq. (12), we can utilize the analytical solution by Baschek et al. (1997a) if we assume that $\chi$ as well as the velocity gradient $w$ are independent of $z$. This assumption implies that the layer considered has to be sufficiently thin, which, however, we do not regard as a severe restriction since any layer can be composed out of thin sub-layers, e.g. by applying the algorithm of Grant and Hunt (cf. Peraiah 1984). In principle, it is possible to allow for a depth dependence of $\chi$ and $w$. Then, however, all expressions would be much more involved, and the expectation values would become functionals of the temperature and pressure distributions. Therefore, should the depth variation of either $\chi$ or $w$ turn out too strong for the thickness of the layer chosen, it seems easier to further subdivide the configuration into thinner layers and then apply the efficient Grant-Peraiah algorithm.

Although the monochromatic extinction coefficient is assumed to be independent of $z$, in a differentially moving medium the Doppler shift $\mathrm{d} \xi=w \mathrm{~d} z$ nevertheless connects frequency with depth.

\subsection{Solution of the comoving-frame transfer equation}

In the moving case, from the solution of Baschek et al. (1997a), the expectation value of the intensity is given by

$$
\begin{aligned}
& \langle I(z, \xi ; w)\rangle=\left\langle\mathcal{I}_{0}(w)\right\rangle \\
& +\frac{1}{w} \int_{\xi-w z}^{\xi}\left\langle\exp \left(-\frac{1}{w} \int_{\eta}^{\xi} \chi(\zeta) \mathrm{d} \zeta\right) \cdot \chi(\eta)\right\rangle S\left(z-\frac{\xi-\eta}{w}, \eta\right) \mathrm{d} \eta \\
& =\left\langle\mathcal{I}_{0}(w)\right\rangle+S(z, \xi) \\
& \quad-S(0, \xi-w z)\left\langle\exp \left(-\frac{1}{w} \int_{\xi-w z}^{\xi} \chi(\zeta) \mathrm{d} \zeta\right)\right\rangle \\
& \quad-\int_{\xi-w z}^{\xi}\left\langle\exp \left(-\frac{1}{w} \int_{\eta}^{\xi} \chi(\zeta) \mathrm{d} \zeta\right)\right\rangle \frac{\mathrm{d} S\left(z-\frac{\xi-\eta}{w}, \eta\right)}{\mathrm{d} \eta} \mathrm{d} \eta
\end{aligned}
$$


where

$\left\langle\mathcal{I}_{0}(w)\right\rangle=\left\langle I(0, \xi-w z ; w) \cdot \exp \left(-\frac{1}{w} \int_{\xi-w z}^{\xi} \chi(\zeta) \mathrm{d} \zeta\right)\right\rangle$

now is the irradiation term for the moving case (cf. Wehrse et al. 1998). As for the static case, the second version (Eq. (14)) has been obtained by partial integration from the first one.

We find that the essential expression to be evaluated in order to discuss the opacity distribution in moving media is

$\left\langle\exp \left(-\frac{1}{w} \int_{\xi-w z}^{\xi} \chi(\zeta) \mathrm{d} \zeta\right)\right\rangle=\mathrm{e}^{-\chi_{\mathrm{c}} \cdot z}\left\langle\mathrm{e}^{-\bar{X}(\xi ; \Delta) \cdot z}\right\rangle$

with

$\Delta=w \cdot z$

and

$$
\begin{aligned}
\bar{X}(\xi ; \Delta) & =\frac{1}{z} \cdot \sum_{l=1}^{L} \frac{1}{w} \int_{\xi-w z}^{\xi} \chi_{l}(\zeta) \mathrm{d} \zeta \\
& =\frac{1}{\Delta} \int_{\xi-\Delta}^{\xi} \sum_{l=1}^{L} \chi_{l}(\zeta) \mathrm{d} \zeta=\frac{1}{\Delta} \int_{\xi-\Delta}^{\xi} X(\zeta) \mathrm{d} \zeta .
\end{aligned}
$$

Thus the crucial expression for the moving case has been cast into exactly the same form as for the conventional static case (see Eq. (9)) with $\bar{X}(\xi ; \Delta)$ replacing $X(\xi)$. Another useful concept when dealing with very many spectral lines is the spectral thickness introduced by Baschek et al. (1997b). In terms of it, the mean of the line extinction coefficient over $\Delta$ reads

$\bar{X}(\xi ; \Delta)=\frac{1}{\Delta} \sum_{l=1}^{L}\left[\psi_{l}(\xi)-\psi_{l}(\xi-\Delta)\right]$,

where $\psi_{l}(\xi)=\int_{\xi_{1}}^{\xi} \chi_{l}(\zeta) \mathrm{d} \zeta$ and $\xi_{1}$ is an arbitrary (logarithmic) reference wavelength.

From Eq. (18) we recognize that $\bar{X}(\xi ; \Delta)$ is the mean line extinction coefficient over the (logarithmic) wavelength interval $(\xi-\Delta, \xi)$ (cf. also the discussion in Wehrse et al. 2000). This two-dimensional set of data can e.g. be calculated from a given deterministic line list in a way completely analogous to the static case. For this, no a priori knowledge of the velocity field is required. Note that the static case is recovered as

$\lim _{\Delta \rightarrow 0} \bar{X}(\xi ; \Delta)=X(\xi)$.

\subsection{Generalized opacity distribution function and the corresponding expectation value}

In analogy to the conventional static case we can now introduce the generalized opacity distribution function $\tilde{p}(\bar{x} ; \xi, \Delta)$ by the probability $\tilde{\mathbb{P}}$ that the mean line extinction coefficient $\bar{X}(\xi ; \Delta)$ in the interval $\Delta$ at $\xi$ has a value between $\bar{x}$ and $\bar{x}+\mathrm{d} \bar{x}$ so that

$\tilde{\mathbb{P}}(\bar{X}(\xi ; \Delta) \in(\bar{x}, \bar{x}+\mathrm{d} \bar{x}))=\tilde{p}(\bar{x} ; \xi, \Delta) \mathrm{d} \bar{x}$

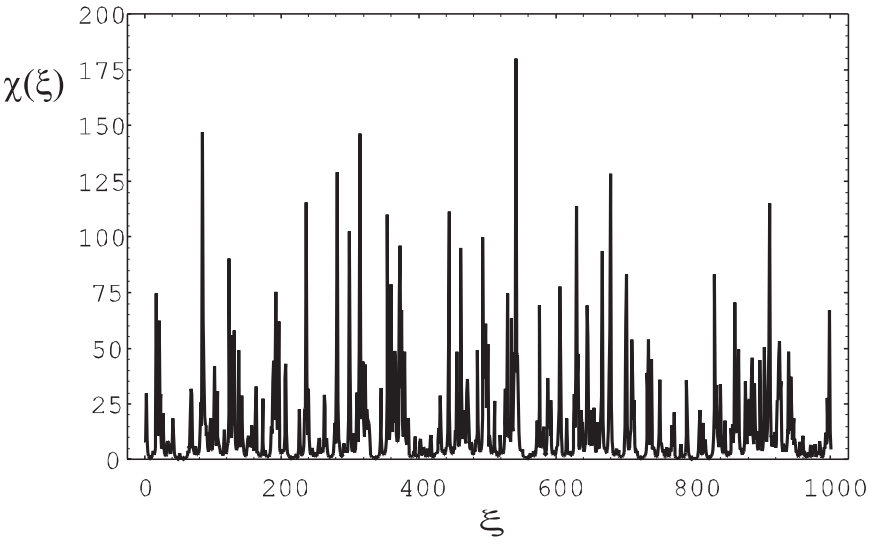

Fig. 1. Typical, simulated deterministic line extinction coefficient $\chi(\xi)$ in arbitrary units, containing $L=400$ lines in the (logarithmic) wavelength range shown. It is assumed that all lines have Lorentzian shapes with the same damping constant $\gamma_{0}$, and that their strengths $A_{l}$ closely follow a power-law with exponent $\alpha=-3 / 2$ (Pareto distribution). For details, see text

with the normalization $\int_{0}^{\infty} \tilde{p}(\bar{x} ; \xi, \Delta) \mathrm{d} \bar{x}=1$. In contrast to the static distribution function, the corresponding probability for the moving case depends on two parameters.

The essential expectation value entering the solution (14) of the transfer equation is

$\left\langle\mathrm{e}^{-\bar{X}(\xi ; \Delta) \cdot z}\right\rangle=\int_{0}^{\infty} \mathrm{e}^{-\bar{x} \cdot z} \tilde{p}(\bar{x} ; \xi, \Delta) \mathrm{d} \bar{x}$.

The further evaluation to obtain the expectation value of the intensity or the flux involves integrations of the expression (22) over the depth variable $z$. We stress that in general $\tilde{p}(\bar{x} ; \xi, \Delta)$ itself depends on $z$ via $\Delta=w z$.

Since $w=$ const has been assumed in the layer considered, $z=\Delta / w$ and $\mathrm{d} z=\mathrm{d} \Delta / w$ holds so that either $\Delta$ or $z$ can be used as integration variable. Furthermore, the Doppler shift can be written as $\mathrm{d} \xi=w \mathrm{~d} z=\mathrm{d} \Delta$.

We note that for the moving case - in contrast to the static case, cf. Eq. (10) - it is not possible to evaluate the expectation value of $\exp (-\bar{X}(\xi ; \Delta) z)($ Eq. $(22))$ as a Laplace transform because $\Delta=w z$ results in a non-linear dependence of $\bar{X}(\xi ; \Delta)$ on $z$.

\section{Examples of opacity distribution functions from a deterministic line list}

The following discussion of the properties of the conventional as well as of the generalized opacity distribution functions is based on an "artificial" deterministic line list which we regard as typical for "real" spectral line lists. From this list, the crucial expression $\bar{X}(\xi ; \Delta)($ Eq. (18)) is then calculated, and from it the generalized opacity distribution function $\tilde{p}(x ; \xi, \Delta)$ according to Eq. (21). The conventional static opacity distribution function is also obtained from $\bar{X}$ as the limit for $\Delta \rightarrow 0$. For convenience, our line list is stochastically generated. We emphasize, however, that - once this simulation of a spectral line list has been obtained - it will subsequently be treated entirely as 

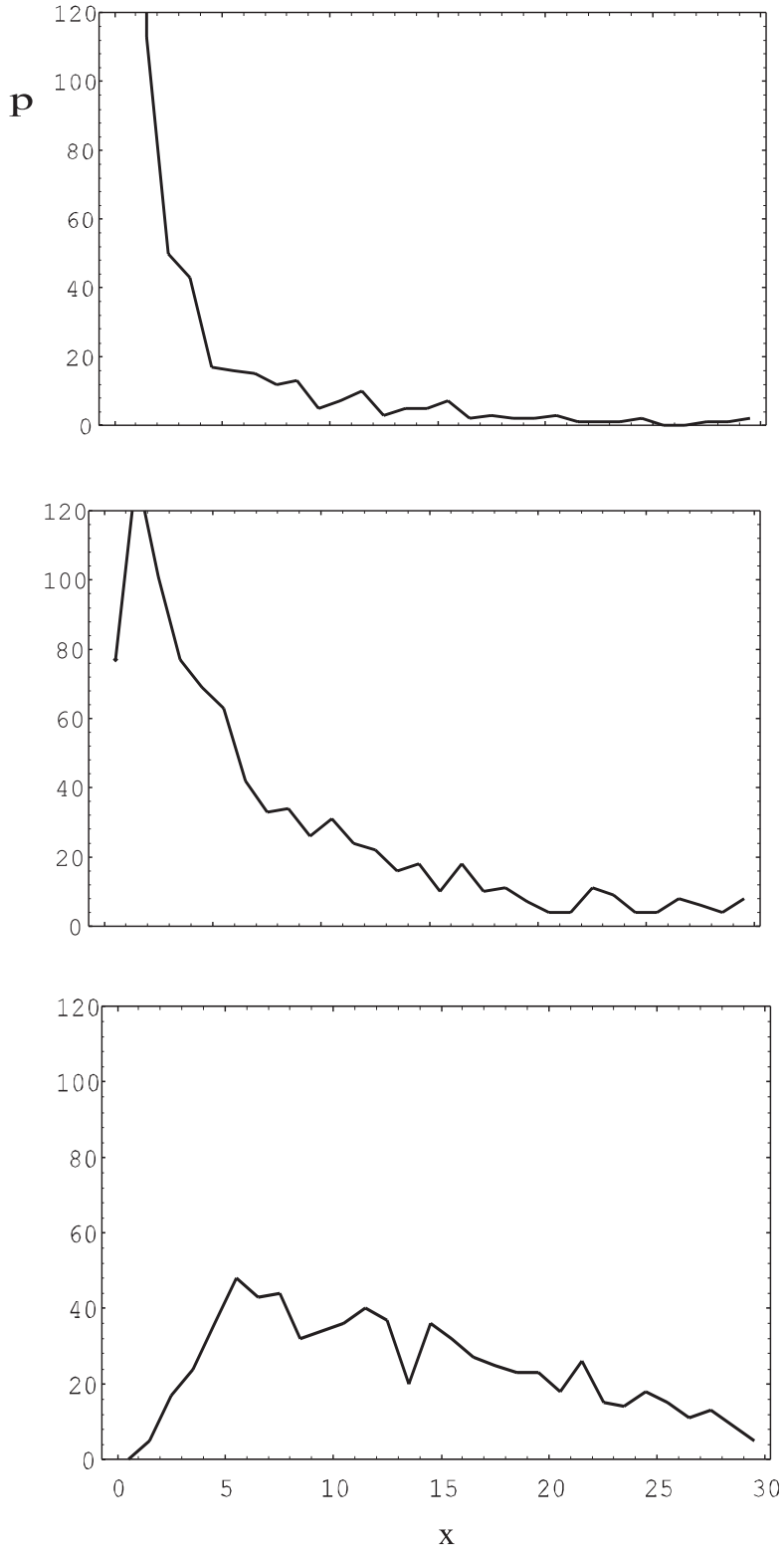

Fig. 2. Static (non-normalized) opacity distribution functions $p(x ; \xi)$ at an arbitrary wavelength $\xi$ for three different values of the total number of lines, $L=100,400$, and 800 (top to bottom) in the interval $\Xi=1000$. The simulation of the underlying extinction coefficient $\chi(\xi)$ is based on the same assumptions as for Fig. 1 with the damping constant assumed to be $\gamma_{0}=10^{-5}$ for all lines

a deterministic list, containing $L$ individual spectral lines in a fixed interval $\Xi$ (in units of the logarithmic wavelength scale $\xi$ ).

The line extinction coefficient $X(\xi)=\sum_{l} \chi_{l}(\xi)$ and its mean $\bar{X}(\xi ; \Delta)$ (Eqs. (1) and (18)) are not only determined by the individual line strengths $A_{l}$, the shape of the lines $\Phi$ and its intrinsic width $\gamma_{l}$ (cf. Eq. (2)), but also by their degree of the "crowding" or overlapping. We thus expect that - besides the average line strength $A_{\mathrm{L}}=\sum_{l} A_{l} / L_{-}$ the number $L$ of spectral lines in the range considered, or, equivalently, the line density

$\varrho_{\mathrm{L}}=L / \Xi$ i.e. the number of lines per unit $\Delta \xi$ interval, will show up as very important parameter of the opacity distribution. The integration interval $\Delta$ (Eq. (17)) entering $\bar{X}(\xi, \Delta)$ implicitly contains the effects of the motions in the medium.

Although to our knowledge a comprehensive investigation of the dependencies of opacity distribution functions on the various parameters is still missing, it is not our aim to provide a full parameter study here. We rather want to demonstrate the main characteristics of the opacity distributions.

\subsection{Generation of a deterministic line list}

For the simulation of the typical deterministic line extinction coefficient $\chi(\xi)$ we assume that the line centers follow an equal probability distribution in the $\xi$ interval and that the strengths $A_{l}$ are described by a log series distribution that is very similar to a power-law (Pareto) distribution of index $\alpha=3 / 2$. We furthermore assume that all lines have Lorentzian profiles with the same intrinsic width (damping constant) $\gamma_{0}$. Details of this stochastic treatment and the underlying assumptions are given in Sects. 5.4 and 7.1 (cf. also Wehrse et al. 1998). A realization of such a $\chi(\xi)$ distribution which serves as the basis for the calculation of our examples is given in Fig. 1: it shows that such a choice of parameters leads to distributions of the extinction coefficients that look quite realistic.

In order to obtain the corresponding generalized opacity distribution function at $\xi$, we suppose that all values of $\tilde{p}(\bar{x} ; \xi, \Delta)$ are actually assumed in an appropriate neighborhood, i.e. we make the ergodic hypothesis, and form for many $\xi_{i}$ of this neighborhood the expression $\bar{X}(\xi ; \Delta)$ according to Eq. (18). We then count the numbers $N_{j}$ for $j=1 \ldots J$ of $\bar{X}\left(\xi_{i} ; \Delta\right)$-values in the intervals $\left[\max \left\{\bar{X}\left(\xi_{i} ; \Delta\right)\right\}-\min \left\{\bar{X}\left(\xi_{i} ; \Delta\right)\right\}\right] /(J-1)$. After normalization, these numbers provide an approximation to the generalized opacity distribution function.

\subsection{Examples of static opacity distributions}

In order to obtain the static case, $\Delta$ is chosen sufficiently small so that the calculated $\bar{X}\left(\xi_{i} ; \Delta\right)$ effectively represent $X\left(\xi_{i}\right) \equiv \bar{X}\left(\xi_{i} ; 0\right)$; in our calculations, $\Delta=0.1$ (i.e $\left.\Delta \ll \gamma_{0}\right)$.

In Fig. 2 the consequences of an increase in the line density are shown for a static medium. It is seen that for a small number $L$ of lines the probability of having small opacities is very large; due to the discretization the value of $X(\xi)=0$ has even the highest probability. High values of $X(\xi)$ have a low probability, as expected. This pattern changes when the number $L$ (or the density $\varrho_{\mathrm{L}}$ ) of the lines is increased: $X(\xi)=0$ then gets the probability zero, but still there is a maximum of $p$ for low values of the extinction coefficient. When the number of lines is increased further the probability distribution gets much broader, and the range of low probability for small $X(\xi)$ value gets wider. 
$\tilde{\mathrm{p}}$
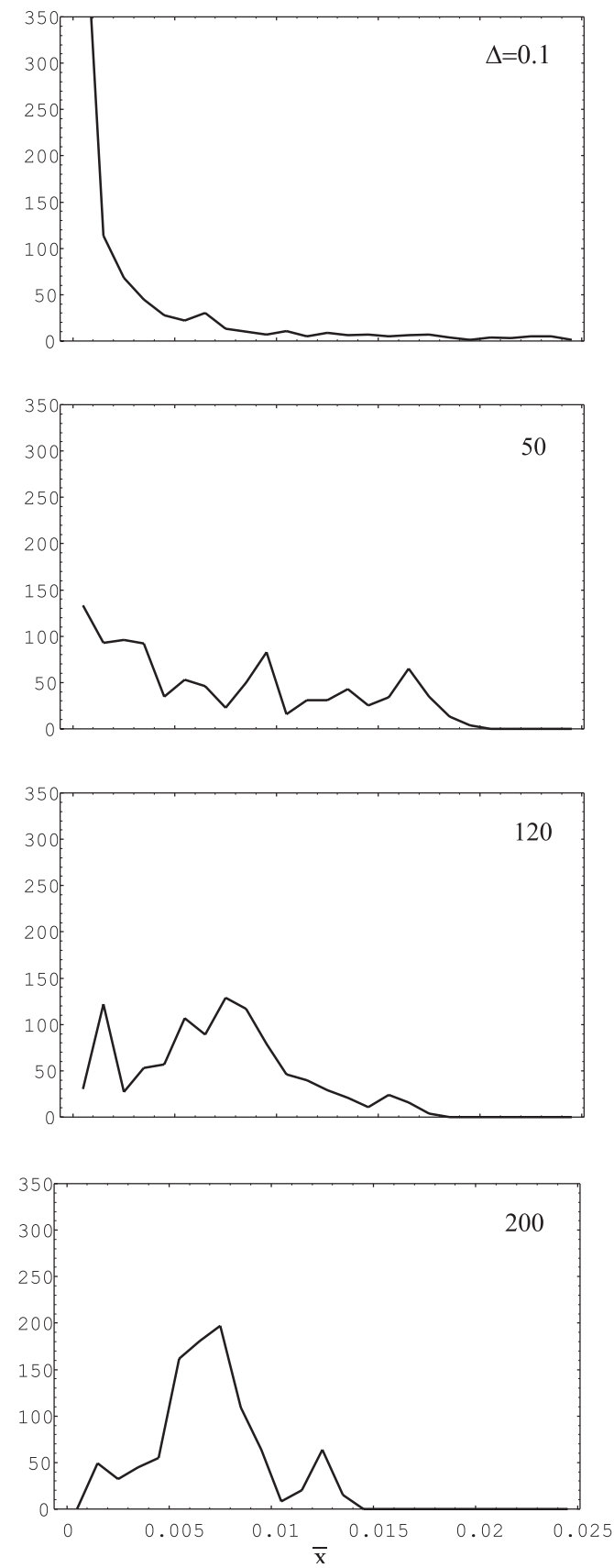
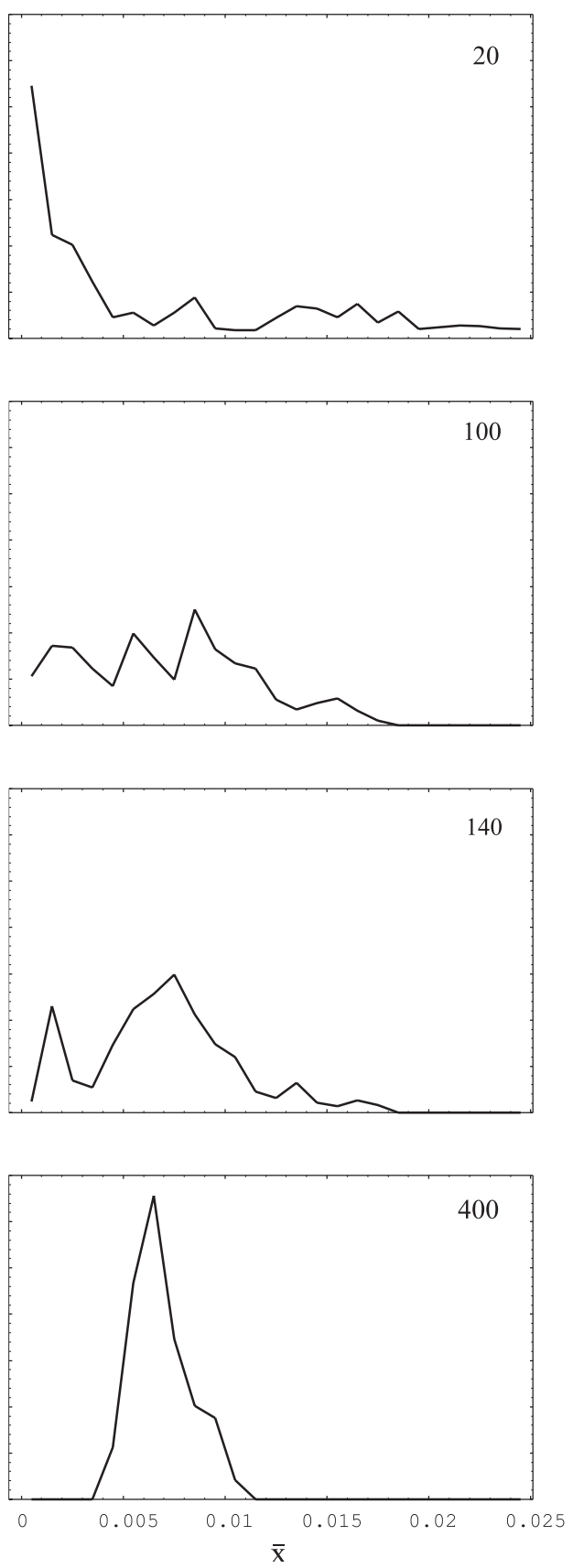

Fig. 3. Generalized (non-normalized) opacity distribution functions $\tilde{p}(\bar{x} ; \xi, \Delta)$ at an arbitrary (logarithmic) wavelength $\xi$ for different values of $\Delta$ (indicated in the upper right of each panel) and a line density $\varrho_{\mathrm{L}}=0.1$. The underlying extinction coefficient $\chi(\xi)$ is simulated with the same assumptions as for Fig. 1; for the calculations, we have expressed the $\xi$ scale in units of the damping constant $\gamma_{0}=1$

We note that the schematic figures of opacity distributions, presented e.g. by Strom \& Kurucz (1966) and Mihalas (1978), refer only to the case of relatively small $L$ or $\varrho_{\mathrm{L}}$.

\subsection{Examples of generalized opacity distributions}

In this section we restrict ourselves to the variation of the generalized opacity distribution function $\tilde{p}$ with $\Delta$ in order to demonstrate - for the first time - the consequences of velocity gradients (Fig. 3): when $\Delta$ is small, the values of $\bar{X}\left(\xi_{i} ; 0\right)$ vary wildly since for small $\Delta$ there is essentially no averaging and $\xi_{i}$ may e.g. be close to the center of a strong line or it may be in an "opacity hole" between lines. Therefore the generalized opacity distribution is rather wide in this case. When $\Delta$ increases, the averaging becomes more and more efficient, and the differences in the extinction coefficients disappear more and more so that $\tilde{p}(\bar{x} ; \xi, \Delta)$ gets more peaked. In the limit of very large $\Delta$ we find a distribution close to a Dirac delta function $\delta(\bar{x}-\langle\bar{x}\rangle)$ with $\langle\bar{x}\rangle=\varrho_{\mathrm{L}} \bar{A}_{\mathrm{L}}$.

Evidently the behavior of $\tilde{p}$ depends also strongly on the variation of the line density $\varrho$ with $\xi$. We expect to 
find a more complicated behavior if $\varrho$ is not constant as had been assumed for Fig. 3 .

\section{Poisson distributed spectral lines}

If one has to deal with a very large number $(L \gg 1)$ of spectral lines it can be advantageous to replace the "deterministic" treatment of the lines by a statistic approach. Wehrse et al. (1998) have shown that the assumption of a Poisson point process is very flexible and well suited to describe the distribution of a large number of lines.

\subsection{Line extinction coefficient}

In order to prepare the transition from the deterministic description to a stochastic one, we first write the contribution of the $L$ lines to the extinction coefficient in the form

$$
X(\xi)=\sum_{l=1}^{L} \chi_{l}(\xi)=\sum_{l=1}^{L} \chi\left(\hat{\xi}_{l}, \vartheta_{l}, \xi-\hat{\xi}_{l}\right)
$$

cf. Eq. (1). Here $\hat{\xi}_{l}$ denotes the center of line $l$, and $\vartheta_{l}=\left\{A_{l}, \gamma_{l}, \Phi_{l}\right\}$ is a multidimensional variable characterizing the properties of the lines. It comprises the line strength $A_{l}$, the intrinsic line width $\gamma_{l}$ and the type of the profile function $\Phi_{l}$. By our notation, we have explicitly introduced the (logarithmic) wavelength position of the line center as an additional variable of $\chi$, and - in order to distinguish it from the "normal" logarithmic wavelength - denote it by $\hat{\xi}_{l}$. In view of the numerous spectral lines we are dealing with, we now regard $\hat{\xi}_{l}$ and $\vartheta_{l}$ as continuous variables and further assume that all lines have the same type of profile function. Then the line extinction coefficient reads

$\chi(\hat{\xi}, \vartheta, \xi-\hat{\xi})=A \cdot \Phi(\hat{\xi}, \gamma, \xi-\hat{\xi})$.

\subsection{Poisson point process: number and density of lines}

We assume that the $\left(\xi_{l}, \vartheta_{l}\right)$ form a Poisson point process (on the direct product set of the total wavelength interval and the set of all possible line parameters). The description by a Poisson point process requires that there are no correlations between the line positions and that the line properties are not correlated. For the definition and details of a Poisson point process we refer to Wehrse et al. (1998).

Let $\varrho(\hat{\xi}, \vartheta) \mathrm{d} \hat{\xi} \mathrm{d} \vartheta$ be the mean number of lines in $\mathrm{d} \hat{\xi} \mathrm{d} \vartheta$, then the "ordinary" mean line density, i.e. the mean number of lines per unit logarithmic wavelength interval at the position $\xi$, is given by

$\varrho(\hat{\xi})=\int_{\Theta} \varrho(\hat{\xi}, \vartheta) \mathrm{d} \vartheta$

and the mean number of all lines is

$\langle L\rangle=\int_{\Theta} \int_{-\infty}^{\infty} \varrho(\hat{\xi}, \vartheta) \mathrm{d} \hat{\xi} \mathrm{d} \vartheta=\int_{-\infty}^{\infty} \varrho(\hat{\xi}) \mathrm{d} \hat{\xi}$.
The total number $L$ of lines is now a random variable, but its expectation value $\langle L\rangle$ differs very little from the actual number of lines since $L$ is very large, i.e. $\langle L\rangle \simeq L$. In the probabilistic description the role of the total number $L$ of the lines is thus taken by $\langle L\rangle$.

In a Poisson point process the total number of lines is Poisson distributed with the probability

$\mathbb{P}\{L=n\}=\frac{\langle L\rangle^{n}}{n !} \mathrm{e}^{-\langle L\rangle}$,

and the number of lines per $\xi$-interval and hence the mean density of lines $\rho(\hat{\xi})$ follow a Poisson distribution as well. For the line positions an equal-probability distribution holds, whereas the distribution of the parameter $\vartheta$ for the line properties can be chosen more or less arbitrarily.

\subsection{Expectation value of $e^{-\bar{X} z}$}

We now turn now to the calculation of the expectation value $\exp (-\bar{X} z)$ which is characteristic for the solution of the radiative transfer equation for the moving case (Eq. (22)). Applying the result by Wehrse et al. (1998, Eqs. (16) and (A6)) for the expectation values of Poisson distributed lines we obtain

$$
\begin{aligned}
\left\langle\mathrm{e}^{-\bar{X}(\xi ; \Delta) \cdot z}\right\rangle & \left\langle\exp \left(-\sum_{l=1}^{L} \frac{1}{w} \int_{\xi-w z}^{\xi} \chi\left(\hat{\xi}_{l}, \vartheta_{l}, \zeta-\hat{\xi}_{l}\right) \mathrm{d} \zeta\right)\right\rangle \\
= & \left\langle\exp \left(-\sum_{l=1}^{L} \frac{1}{\Delta} \int_{\xi-\Delta}^{\xi} \chi\left(\hat{\xi}_{l}, \vartheta_{l}, \zeta-\hat{\xi}_{l}\right) \mathrm{d} \zeta \cdot z\right)\right\rangle \\
= & \exp \left(\int_{\Theta}^{\infty} \int_{-\infty}^{\infty} \varrho(\hat{\xi}, \vartheta)\right. \\
& \left.\times\left\{\exp \left(-\frac{z}{\Delta} \int_{\xi-\Delta}^{\xi} \chi(\hat{\xi}, \vartheta, \zeta-\hat{\xi}) \mathrm{d} \zeta\right)-1\right\} \mathrm{d} \hat{\xi} \mathrm{d} \vartheta\right)
\end{aligned}
$$

An equivalent expression can be obtained by introducing the abbreviation by Wehrse et al. (1998) for the line contribution to the expectation value for Poisson distributed lines,

$\Omega(\xi, \eta ; w)=\left\langle\exp \left(-\frac{1}{w} \sum_{l=1}^{L} \int_{\eta}^{\xi} \chi\left(\hat{\xi}_{l}, \vartheta_{l}, \zeta-\hat{\xi}_{l}\right) \mathrm{d} \zeta\right)\right\rangle$,

so that

$\left\langle\mathrm{e}^{-\bar{X}(\xi ; w z) \cdot z}\right\rangle=\Omega(\xi, \xi-w z ; w)$.

The expectation value of $\exp (-X z)$ for the static case can be obtained as the limit $w, \Delta \rightarrow 0$ (cf. Eq. (20)) yielding

$$
\begin{aligned}
& \left\langle\mathrm{e}^{-X(\xi) \cdot z}\right\rangle=\Omega(\xi, \xi ; 0) \\
& =\left\langle\exp \left(-\sum_{l=1}^{L} \chi\left(\hat{\xi}_{l}, \vartheta_{l}, \xi-\hat{\xi}_{l}\right) \cdot z\right)\right\rangle \\
& =\exp \left[\int_{\Theta}^{\infty} \int_{-\infty}^{\infty} \varrho(\hat{\xi}, \vartheta)\left(\mathrm{e}^{-\chi(\hat{\xi}, \vartheta, \xi-\hat{\xi}) \cdot z}-1\right) \mathrm{d} \hat{\xi} \mathrm{d} \vartheta\right] .
\end{aligned}
$$




\subsection{Basic functions of the Poisson point process}

As can be seen from Eq. (29), the characteristic expectation value occurring in the Poisson point process is a functional of two basic functions:

1. the line extinction coefficient $\chi(\hat{\xi}, \vartheta, \xi-\hat{\xi})($ Eq. $(25))$ and

2. the distribution $\varrho(\hat{\xi}, \vartheta)$ of the lines in $(\hat{\xi}, \vartheta)$-space.

These two functions also determine the generalized opacity distribution function (cf. Sect. 6).

Of course, for any actual calculation the basic functions have to be specified. First, in most cases we may assume that in each spectral interval $\mathrm{d} \hat{\xi}$ the parameter $\vartheta$, describing the line properties, is independent of the mean line density $\varrho(\hat{\xi})($ Eq. $(26))$ so that

$\varrho(\hat{\xi}, \vartheta)=\varrho(\hat{\xi}) \cdot f(\vartheta)$,

where $f(\vartheta)$ is the distribution function of $\vartheta$, normalized according to $\int_{\Theta} f(\vartheta) \mathrm{d} \vartheta=1$.

A further reasonable assumption is, that all spectral lines have the same intrinsic shape or profile function $\Phi$ and the same intrinsic width $\gamma_{0}$.

Finally, with all these assumptions, the two basic functions of the Poisson point process become

$$
\begin{aligned}
& \chi(\hat{\xi}, \vartheta, \xi-\hat{\xi})=A \cdot \Phi\left(\hat{\xi}, \gamma_{0}, \xi-\hat{\xi}\right) \text { and } \\
& \varrho(\hat{\xi}, \vartheta) \cdot \delta\left(\gamma-\gamma_{0}\right)=\varrho(\hat{\xi}, A)=\varrho(\hat{\xi}) \cdot f(A) \cdot \delta\left(\gamma-\gamma_{0}\right)
\end{aligned}
$$

where the line strength distribution is normalized according to $\int_{A_{1}}^{A_{2}} f(A) \mathrm{d} A=1$. Thus $\varrho(\hat{\xi}, \vartheta)$ is determined solely by the product of the mean line density and line strength distribution.

To summarize, for any application the quantities yet to be specified are

the profile function $\Phi$ and its intrinsic width $\gamma_{0}$, the line density $\varrho(\hat{\xi})$, and

the distribution $f(A)$ of the line strengths.

In our numerical examples which will be given in Sect. 7, we adopt these assumptions, and in particular we consider Lorentz profiles with the same damping constant $\gamma_{0}$ for all lines, and with the distribution $f(A)$ of their strengths following a power-law or Pareto distribution.

After this short introductory overview over the statistic description of the spectral lines in terms of a Poisson point process, we now turn to the derivation of the corresponding generalized opacity distribution.

\section{Generalized opacity distribution function for Poisson distributed lines}

To derive the generalized opacity distribution function $\tilde{p}$ for lines described by the Poisson point process, we start by applying Fourier's integral theorem to $\tilde{p}$ :

$$
\begin{aligned}
\tilde{p}(\bar{x} ; \xi, \Delta) & =\mathcal{F}_{\bar{x}}^{-1}\left\{\mathcal{F}_{k}\{\tilde{p}\}\right\} \\
& =\frac{1}{2 \pi} \int_{-\infty}^{\infty} \mathrm{e}^{i k \bar{x}}\left[\int_{-\infty}^{\infty} \mathrm{e}^{-i k \bar{y}} \tilde{p}(\bar{y} ; \xi, \Delta) \mathrm{d} \bar{y}\right] \mathrm{d} k
\end{aligned}
$$

where

$$
\mathcal{F}_{k}\{\tilde{p}\} \equiv \mathbb{F}(k ; \xi, \Delta)=\int_{-\infty}^{\infty} \mathrm{e}^{-i k \bar{y}} \tilde{p}(\bar{y} ; \xi, \Delta) \mathrm{d} \bar{y}
$$

is the Fourier transform and $\mathcal{F}_{\bar{x}}^{-1}$ is its inverse; here $k$ is real, and $\tilde{p}$, of course, is a real function with $\tilde{p} \geq 0$. Since on the one hand $\bar{x}$ (or $\bar{y}$ ) are positive quantities and on the other hand negative values occur in the Fourier integrals, we formally set $\tilde{p}=0$ for $\bar{x}, \bar{y}<0$. Note that here the arguments $\xi$ and $\Delta$ of $\tilde{p}$ occur only as parameters.

In analogy to Eq. (22), the inner Fourier transform can be regarded as the expectation value

$\left\langle\mathrm{e}^{-\bar{X}(\xi ; \Delta) \cdot i k}\right\rangle=\int_{-\infty}^{\infty} \mathrm{e}^{-\bar{x} \cdot i k} \tilde{p}(\bar{x} ; \xi, \Delta) \mathrm{d} \bar{x}$

so that

$\tilde{p}(\bar{x} ; \xi, \Delta)=\frac{1}{2 \pi} \int_{-\infty}^{\infty} \mathrm{e}^{i k \bar{x}} \cdot\left\langle\mathrm{e}^{-\bar{X}(\xi ; \Delta) \cdot i k}\right\rangle \mathrm{d} k$.

This pair of relationships is equivalent to Eq. (35).

We note that in probability theory $\langle\exp (i k X)\rangle$ is the characteristic function of the probability density $p(x)$ of $X$, and it is well known that $p(x)$ is the Fourier transform of the characteristic function (e.g. Feller 1966; Reichl 1980).

For a Poisson distribution $p(X=n)=\lambda^{n} \mathrm{e}^{-\lambda} / n$ !, the characteristic function is $\langle\exp (i k X)\rangle=\exp \left[\lambda\left(\mathrm{e}^{i k}-1\right)\right]$ (Fisz 1978). This basic structure can also be recognized in our expression (32) although here we do not directly consider a Poisson distribution, but rather deal with the distribution of the wavelength-integrated line extinction coefficients $\bar{X}(\xi ; \Delta)$ where it is only the total number of lines which obeys a Poisson distribution (Eq. (28)).

We evaluate the expectation value (37) for a line distribution obeying a Poisson point process from Eq. (29) with the assumptions (34) by setting $z=i k$ and obtain

$$
\begin{aligned}
& \left\langle\mathrm{e}^{-\bar{X}(\xi ; \Delta) \cdot i k}\right\rangle \\
& =\exp \left(\int_{A_{1}}^{A_{2}} \int_{-\infty}^{\infty} \varrho(\hat{\xi}, A)\left\{\mathrm{e}^{-i k A U}-1\right\} \mathrm{d} \hat{\xi} \mathrm{d} A\right)
\end{aligned}
$$

where we have introduced the abbreviation

$$
U \equiv U\left(\hat{\xi}, \xi, \Delta, \gamma_{0}\right)=\frac{1}{\Delta} \int_{\xi-\Delta}^{\xi} \Phi\left(\hat{\xi}, \gamma_{0}, \zeta-\hat{\xi}\right) \mathrm{d} \zeta
$$

We point out that the expression $A \cdot U\left(\hat{\xi}, \xi, \Delta, \gamma_{0}\right)$, occurring for Poisson distributed lines, is not to be confused with $\bar{X}(\xi ; \Delta)$ defined in Eq. (18) although both formally are similar. $U$ is not of the type of a mean value such as $\bar{X}$, but rather is defined as the integral over the single line which is characteristic for the Poisson point process. The limit of $U$ for $\Delta \rightarrow \infty$ is zero. In fact, Eq. (39) establishes the connection between $U$ and $\bar{X}$. 
According to Eq. (38), the generalized opacity distribution function is given by

$$
\begin{aligned}
& \tilde{p}(\bar{x} ; \xi, \Delta) \\
& =\frac{1}{2 \pi} \int_{-\infty}^{\infty} \exp [i k \bar{x} \\
& \left.\quad+\int_{A_{1}}^{A_{2}} \int_{-\infty}^{\infty} \varrho(\hat{\xi}, A)\left\{\mathrm{e}^{-i k A U}-1\right\} \mathrm{d} \hat{\xi} \mathrm{d} A\right] \mathrm{d} k \\
& =\frac{1}{\pi} \int_{0}^{\infty} \exp \left[\int_{A_{1}}^{A_{2}} \int_{-\infty}^{\infty} \varrho(\hat{\xi}, A)\{\cos (k A U)-1\} \mathrm{d} \hat{\xi} \mathrm{d} A\right] \\
& \quad \times \cos \left\{k \bar{x}-\int_{A_{1}}^{A_{2}} \int_{-\infty}^{\infty} \varrho(\hat{\xi}, A) \sin (k A U) \mathrm{d} \hat{\xi} \mathrm{d} A\right\} \mathrm{d} k .
\end{aligned}
$$

This is the central connection of the opacity distribution function to the Poisson statistics in the case of a moving medium.

In order to further evaluate the expressions (41) and (42), we have to specify the two basic functions (34) for Poisson distributed lines. The generalized opacity distribution function $\tilde{p}(\bar{x} ; \xi, \Delta)$ can then be calculated by means of the fast Fourier transform (see Appendix).

\subsection{Static limit}

The conventional opacity distribution function $p(x ; \xi)$ for Poisson distributed lines is obtained from Eqs. $(41,42)$ as the limit for $\Delta \rightarrow 0$ :

$$
\begin{aligned}
& p(x ; \xi) \\
& =\frac{1}{2 \pi} \int_{-\infty}^{\infty} \exp [i k x \\
& \left.\quad+\int_{A_{1}}^{A_{2}} \int_{-\infty}^{\infty} \varrho(\hat{\xi}, A)\left\{\mathrm{e}^{-i k A \Phi\left(\hat{\xi}, \gamma_{0}, \xi-\hat{\xi}\right)}-1\right\} \mathrm{d} \hat{\xi} \mathrm{d} A\right] \mathrm{d} k \\
& =\frac{1}{\pi} \int_{0}^{+\infty} \exp \left[\int_{A_{1}}^{A_{2}} \int_{-\infty}^{\infty} \varrho(\hat{\xi}, A)\right. \\
& \left.\quad \times\left\{\cos \left(k A \Phi\left(\hat{\xi}, \gamma_{0}, \xi-\hat{\xi}\right)\right)-1\right\} \mathrm{d} \hat{\xi} \mathrm{d} A\right] \\
& \quad \times \cos \left(k x-\int_{A_{1}}^{A_{2}} \int_{-\infty}^{\infty} \varrho(\hat{\xi}, A)\right. \\
& \left.\quad \times \sin \left(k A \Phi\left(\hat{\xi}, \gamma_{0}, \xi-\hat{\xi}\right)\right) \mathrm{d} \hat{\xi} \mathrm{d} A\right) \mathrm{d} k
\end{aligned}
$$

where we have used from Eq. (40) that

$\lim _{\Delta \rightarrow 0} U\left(\hat{\xi}, \xi, \Delta, \gamma_{0}\right)=\Phi\left(\hat{\xi}, \gamma_{0}, \xi-\hat{\xi}\right)$.

Here the static opacity distribution function $p(x ; \xi)$ for Poisson distributed lines is obtained by the Fourier integral of the corresponding expectation value $\langle\exp (-X \cdot z)\rangle$ (Eq. (32)). Conversely, for the static case, the expectation value can be recovered from the opacity distribution function by applying a Laplace transform to $p(x ; \xi)$ according to Eq. (10). It may be instructive to give some details of how this inverse step is achieved: by inserting $p(x ; \xi)$ from Eqs. (43) into (10) we get

$\left\langle\mathrm{e}^{-X \cdot z}\right\rangle=\frac{1}{2 \pi} \int_{0}^{\infty} \mathrm{e}^{-x z} \int_{-\infty}^{\infty} \mathrm{e}^{i k x} C(k) \mathrm{d} k \mathrm{~d} x$

with

$$
\begin{aligned}
& C(k)= \\
& \exp \left(\int_{A_{1}}^{A_{2}} \int_{-\infty}^{\infty} \varrho(\hat{\xi}, A)\left\{\mathrm{e}^{-i k A \Phi\left(\hat{\xi}, \gamma_{0}, \xi-\hat{\xi}\right)}-1\right\} \mathrm{d} \hat{\xi} \mathrm{d} A\right) .
\end{aligned}
$$

Performing the integration over $x$ first, results in

$\int_{0}^{\infty} \mathrm{e}^{(i k-z) x} d x=\frac{1}{z-i k}=\frac{i}{k+i z}$

( $k$ real, $z>0$ ). The remaining integral over $k$ along the real axis is evaluated by application of the residue theorem in the complex $k$-plane. To this purpose,

$\frac{C(k)}{k+i z}=\sum_{n=-\infty}^{\infty} a_{n} \cdot\left(k-k_{0}\right)^{n}$

is expanded as a Laurent series around $k_{0}=-i z$, where $a_{-1}=C(-i z)$. This finally leads to the expectation value given by Eq. (32).

We note that, in contrast to the static case, however, the analogous transformation for the moving case cannot be carried out since $\Delta=w z$ also contains $z$.

The fact that the expectation value $\langle\exp (-X z)\rangle$ can be expressed as the Laplace transform of $p(x ; \xi)$ allows us - again for the static case only - to express also the characteristic function of $p(x ; \xi)$ either as the Laplace or as the (inverse) Fourier transform of $p$ : Since $\mathbb{L}(z ; \xi)$ in Eq. (10) can be defined for all complex $z$ with $\operatorname{Re}(z) \geq 0$, we may formally choose the purely imaginary value $z=$ $-i k$ to arrive at

$$
\begin{aligned}
\mathcal{L}_{-i k}\{p\} & =\int_{0}^{\infty} \mathrm{e}^{i k x} p(x ; \xi) \mathrm{d} x=\int_{-\infty}^{\infty} \mathrm{e}^{i k x} p(x ; \xi) \mathrm{d} x \\
& =2 \pi \mathcal{F}_{k}^{-1}\{p\}=\left\langle\mathrm{e}^{i k X}\right\rangle .
\end{aligned}
$$

Note that the "single line" which appears in Eqs. (43) and (44) as a consequence of the assumed Poisson point process may be regarded as the statistical analogue of the "fat line" introduced in the pioneering work by Labs (1951).

\section{Examples of opacity distribution functions for Poisson distributed spectral lines}

In our following examples of opacity distribution functions with the formalism of the Poisson point process no attempt is made to systematically cover the entire range of the relevant parameters. Instead, our specific assumptions and choice of the parameters aim at the presentation of a few typical cases which allows to discuss the fundamental dependence of the opacity distribution on the most important parameters (and which furthermore requires not too large an effort for the numerical calculation). 

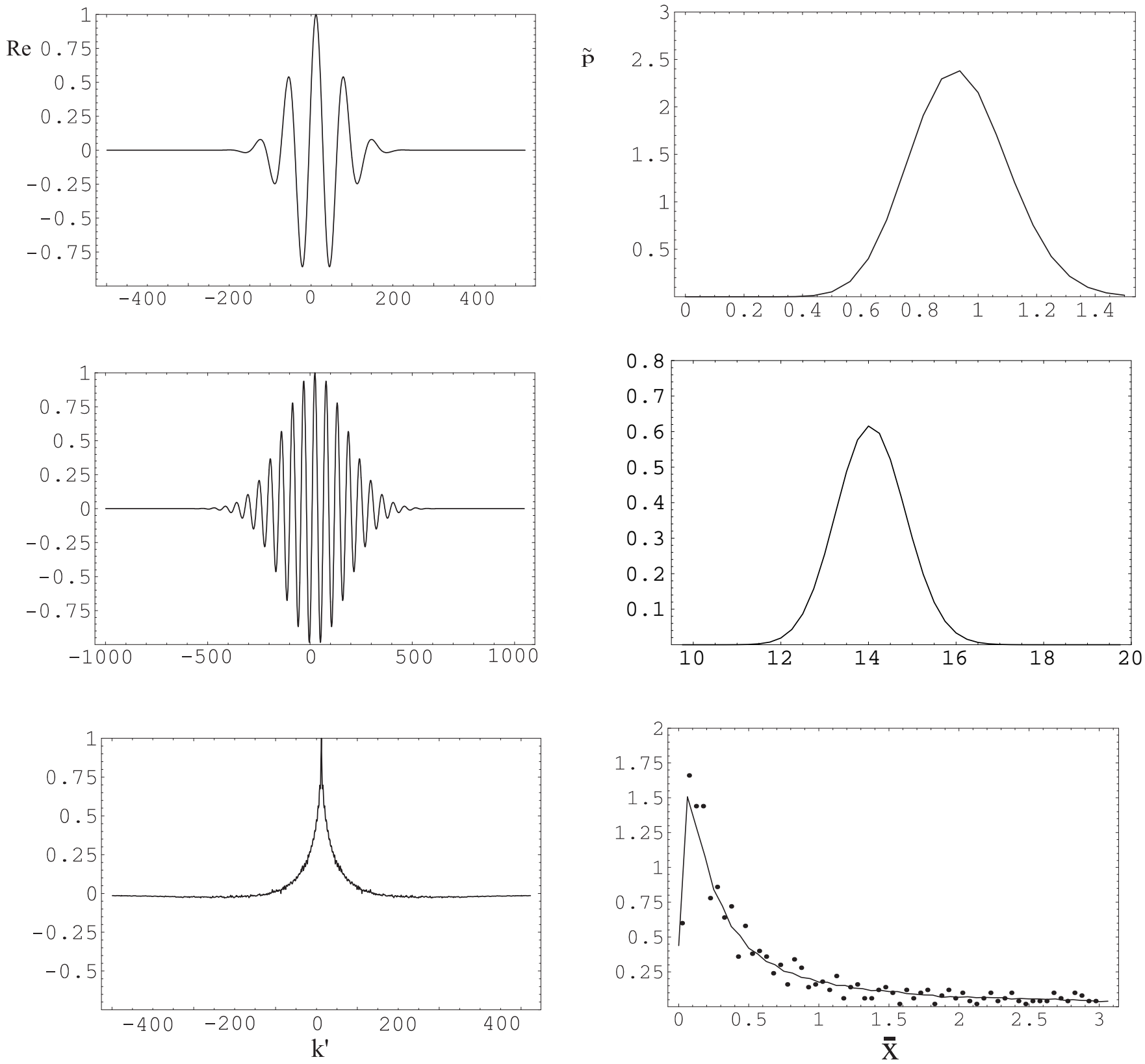

Fig. 4. Generalized opacity distribution functions $\tilde{p}(\bar{x} ; \xi, \Delta)$ (right column) and the real parts of their corresponding expectation values, $\operatorname{Re}(\langle\exp (-\bar{X}(\xi ; \Delta) \cdot i k)\rangle)$ (left column), for Poisson distributed lines at an arbitrary (logarithmic) wavelength $\xi$. Shown are examples for three combinations of the line density $\varrho$ and the averaging parameter $\Delta:(10,100),(100,100)$, and $(1,0.1)$ (top to bottom), where $k=a k^{\prime}$ with $a=64,512$, and 64 (top to bottom). For comparison, we have added (as dots) in the lower right panel values for the generalized opacity distribution function calculated according to the procedure described in Sect. 3 . The lines have the same statistical properties in both cases. Note that the small wiggles in the lower left panel result from inaccuracies in the $\hat{\xi}$-integration (cf. Eq. (41)) as a consequence of a highly oscillatory behavior of the integrand

\subsection{Additional assumptions for the examples}

For all examples given here, we adopt the assumptions given in Eq. (34). In addition we specifically assume that all spectral lines are described by a Lorentz profile with the damping constant $\gamma_{0}$ (in logarithmic frequency units),

$\Phi\left(\hat{\xi}, \gamma_{0}, \xi-\hat{\xi}\right)=\frac{A}{\pi} \frac{\gamma_{0} / 2}{(\xi-\hat{\xi})^{2}+\left(\gamma_{0} / 2\right)^{2}}$.
Furthermore we adopt a power-law $f(A) \propto A^{-\alpha}$ in the range from $A_{1}$ to $A_{2}$ for the distribution of the line strengths $A$ with the particular index $\alpha=3 / 2$,

$$
f(A) \mathrm{d} A=c_{A} A^{-3 / 2} \mathrm{~d} A \quad \text { with } \quad c_{A}=\frac{1}{2} \frac{\sqrt{A_{1} A_{2}}}{\sqrt{A_{2}}-\sqrt{A_{1}}}
$$

an assumption which is in accord with theoretical and empirical results of atomic spectroscopy (see e.g. Wehrse et al. 1998). 
In probability theory, the probability density $f(x)=$ $(\alpha-1) / x_{0} \cdot\left(x / x_{0}\right)^{-\alpha}$ for $x>x_{0}$, and $=0$ for $x \leq x_{0}$, with $\alpha>1$ is known as Pareto distribution (see e.g. Fisz 1978), so that Eq. (52) represents a trimmed Pareto distribution with $\alpha=3 / 2$.

For $\alpha=3 / 2$, the mean line strength,

$\bar{A}=\int_{A_{1}}^{A_{2}} A f(A) \mathrm{d} A=\sqrt{A_{1} A_{2}}$,

is the harmonic mean of the smallest and largest line strength considered. We note that a useful simplified version of the line strength distribution (52) can be obtained in the limiting case of a small range in $A$, i.e. for $\Delta A=A_{2}-A_{1} \ll \bar{A}$ :

$f(A) \mathrm{d} A \simeq \frac{1}{\Delta A} \cdot\left(\frac{A}{\bar{A}}\right)^{-3 / 2} \mathrm{~d} A$,

where $A_{1,2} \simeq \bar{A} \mp \Delta A / 2$.

\subsection{Opacity distribution function for a line strength distribution $\propto A^{-3 / 2}$}

For the case that $f(A)$ is presented by the trimmed Pareto distribution (52) and Lorentz line profiles (51) are considered, the first steps in the calculation of the generalized opacity distribution function can be carried out analytically. We get for the expectation value (Eq. (39))

$$
\left\langle\mathrm{e}^{-\bar{X}(\xi ; \Delta) \cdot i k}\right\rangle=\exp \left(\int_{-\infty}^{\infty} \varrho(\hat{\xi}) Y(k, U) \mathrm{d} \hat{\xi}\right)
$$

where

$$
\begin{aligned}
Y(k, U) & =\exp \left(c_{A} \int_{A_{1}}^{A_{2}} A^{-3 / 2}(\exp (-A \cdot i k U)-1) \mathrm{d} A\right) \\
= & 2 c_{A} \cdot\left(\frac{\mathrm{e}^{-A_{1} \cdot i k U}-1}{\sqrt{A_{1}}}-\frac{\mathrm{e}^{-A_{2} \cdot i k U}-1}{\sqrt{A_{2}}}\right. \\
& \left.+\sqrt{k} \sqrt{\pi i U}\left[\operatorname{Erf}\left(\sqrt{k} \sqrt{i A_{1} U}\right)-\operatorname{Erf}\left(\sqrt{k} \sqrt{i A_{2} U}\right)\right]\right) .
\end{aligned}
$$

For a Lorentz profile with a damping constant $\gamma_{0}$, the integral in Eq. (40) becomes

$U=\frac{1}{\pi \Delta} \cdot\left(\arctan \frac{\xi-\hat{\xi}}{\gamma_{0} / 2}-\arctan \frac{(\xi-\hat{\xi})-\Delta}{\gamma_{0} / 2}\right)$.

In the final step, the generalized opacity distribution function $\tilde{p}(\bar{x} ; \xi, \Delta)$ is obtained by (numerical) integration over $k$ according to Eqs. (41) or (42).

\subsection{Examples}

For all examples of calculations of generalized opacity distribution functions $\tilde{p}(\bar{x} ; \xi, \Delta)$ for Poisson distributed lines we keep $A_{1}$ and $A_{2}$, and hence also $c_{A}$ and $\bar{A}$ fixed, and vary only the line density $\varrho(\hat{\xi})$ which we for simplicity - without substantial loss of insight - assume to be independent of $\xi$, i.e. $\varrho(\xi)=\varrho_{0}$ and the damping constant $\gamma_{0}$.

In Fig. 4 we present $\operatorname{Re}(\langle\exp (-\bar{X}(\xi ; \Delta) \cdot i k)\rangle)$ and the Fourier transform of the full, i.e. complex expectation value which is the generalized opacity distribution function $\tilde{p}(\bar{x} ; \xi, \Delta)$. The values of $\Delta$ are 100,100 , and 0.1 , and those of the line density $\varrho_{0}$ are 10, 100, and 1 . For all examples; the damping constant is $\gamma_{0}=0.1$, and the line strengths are allowed to vary over a small range only so that we have essentially for all lines $A=1$. In test calculations some wider ranges in $A$ have been adopted as well, demonstrating that the upper and lower bounds, $A_{1}$ and $A_{2}$, have such a strong influence on the resulting distribution function that the parameters for the integrations (e.g. the step widths) had to be readjusted.

It is evident that the frequency of the oscillation in $\operatorname{Re}(\langle\exp (-\bar{X}(\xi ; \Delta) \cdot i k)\rangle)$ determines the position of the peak of the generalized opacity distribution function $\tilde{p}(\bar{x} ; \xi, \Delta)$, and that the fall-off of the amplitudes controls the width of $\tilde{p}$. Equation (42) reveals that that the falloff is governed by $\iint \varrho\{\cos (k A U)-1\} \mathrm{d} \hat{\xi} \mathrm{d} A$, and the frequency by $\iint \varrho \sin (k A U) \mathrm{d} \hat{\xi} \mathrm{d} A$. Due the complicated nature of the integrals, the detailed dependence on the line densities and line parameters is intricate, and its discussion is therefore beyond the scope of this paper.

As is seen from the lower right panel of Fig. 4, there is an excellent agreement between the opacity distribution determined by means of a Poisson point process and that calculated directly from averaged extinction coefficients, i.e. from simulations according to Sect. 3. We therefore consider both ways essentially as equivalent; the latter just has some computational advantages whereas the former allows a much deeper insight.

\section{Discussion}

By the algorithms introduced in this paper, new ways are added to the possibilities of treating many lines in radiative transfer problems. First, in static media the opacity distribution function can be calculated by means of a Poisson point process. Secondly, two ways are suggested for the application to differentially moving media where a generalized opacity distribution function can be derived either directly from running averages of the absorption coefficient or from the Poission point process. This implies in particular that there are now methods available that allow an equally efficient treatment of radiation fields in static and in differentially moving configurations.

The resulting logical dependencies are summarized in Fig. 5. It is seen that all cases rely on a proper line list and that then there is a variety of ways to proceed. The way chosen in an actual problem has then to be determined by the particular circumstances such as e.g. the availability of large computers, the programming effort to be invested, or the frequency resolution needed. Note in particular that the static case can always be regained as the limit $w \rightarrow 0$.

It is evident that in particular the assumption of a Poisson point process not only speeds up strongly the 


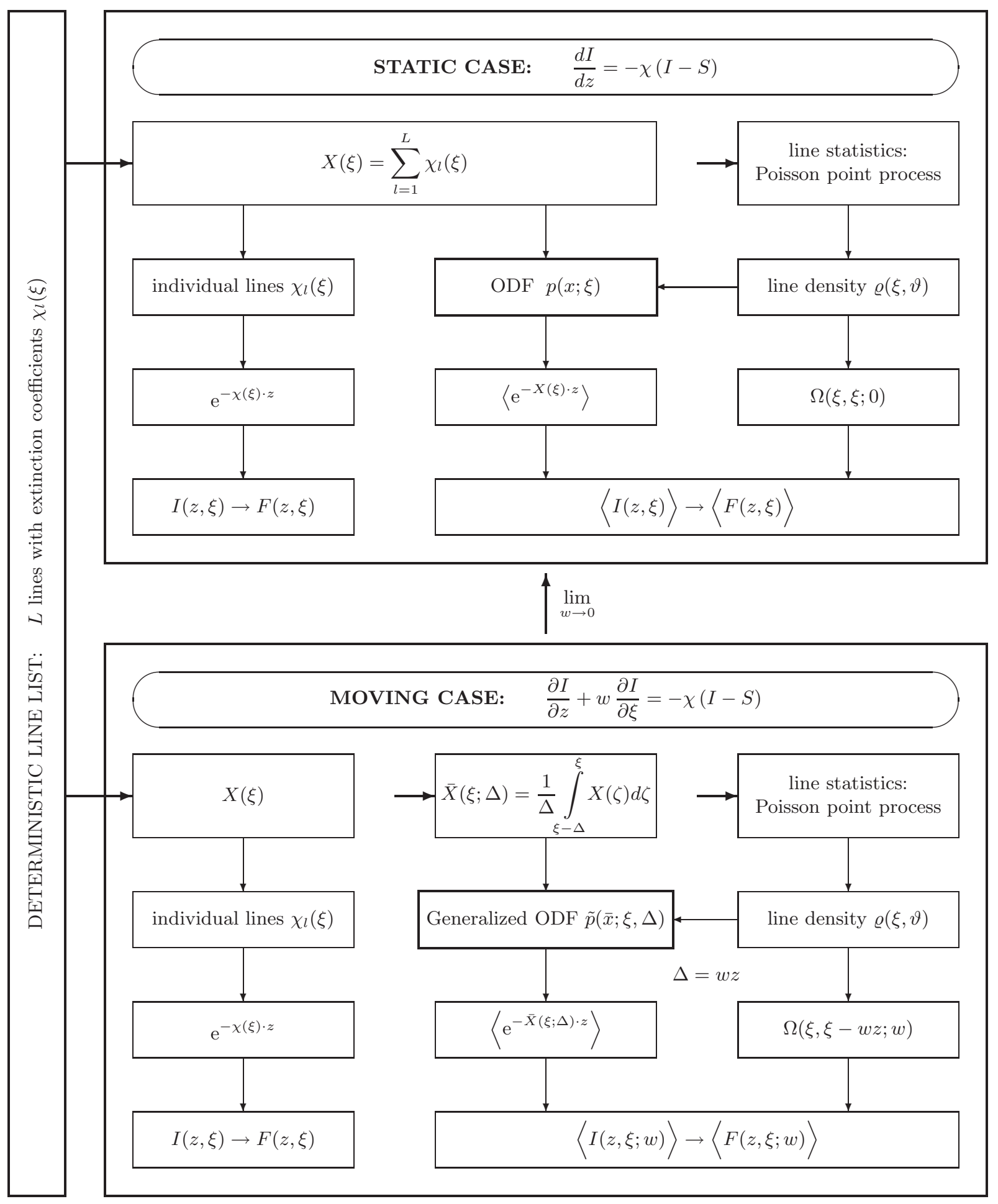

Fig. 5. Basic expressions and interrelations for the Generalized ODF for a differentially moving plane-parallel layer and the corresponding conventional static ODF (opacity distribution function)

modeling of radiation fields but it also provides possibilities to solve problems that have been inaccessible up to now as e.g. the influence of very many, very weak lines. However, it has also to be acknowledged that this paper provides only the basics for the statistical methods.
Subsequent steps must comprise investigations (i) on the dependencies of the shape of the distribution function on the various parameters, (ii) on possibilities of simplifying the ODF without significant loss of accuracy, e.g. as the representation by a normal distribution as is suggested by 
the shapes shown in Figs. 2 to 4, and by the central limit theorem, (iii) on alternative algorithms for the determination of the distribution function that might not only be faster and avoid the difficult integrations (cf. Eqs. (41), (42)) but that also allow a more complete insight such as e.g. a cumulant expansion (cf. Stuart \& Ord 1987) in which correlations would show up immediately, and (iv) on the proper densities of line strengths, shapes, and spacing as functions of element abundances, temperatures and pressures. Further studies should be devoted to the extension to scattering lines and to NLTE conditions (e.g. by the use of Wigner matrices).

Acknowledgements. We are indebted to G. V. Efimov, W. Kalkofen, A. Mikelic, and G. Shaviv for many helpful discussions. This work has been supported in part by the DFG (Sonderforschungsbereich 359/C2).

\section{Appendix: Determination of the ODF by fast Fourier transformation}

The actual calculation of the generalized opacity distribution function $\tilde{p}(\bar{x} ; \xi, \Delta)$ from Eq. (41) by means of the fast Fourier transformation is intricate and requires special care. It is best performed in the following way:

1. The integral

$$
\int_{A_{1}}^{A_{2}} \int_{-\infty}^{\infty} \varrho(\hat{\xi}, A)\left\{\exp \left(-i k A U\left(\hat{\xi}, \xi, \Delta, \gamma_{0}\right)\right)-1\right\} \mathrm{d} \hat{\xi} \mathrm{d} A
$$

is decomposed into its real and imaginary parts whenever possible;

2. the integrations over $\zeta$ and $A$ are performed analytically (e.g. for Lorentz or Gauss profiles);

3. the integrations over $\hat{\xi}$ are done numerically for $2^{n}$ values of $k$, where $n$ and $\Delta k$ have to be chosen in such a way that the variations of the integrals is sufficiently well reproduced and their values are sufficiently negative so that the corresponding exponentials for the largest $k$ values are close to zero;

4. an array $Q$ is formed of these exponentials and subsequently an array $S$ consisting of $Q$ and the transposed conjugate complex of $Q$;

5. the array $S$ is fast-Fourier-transformed to give a discrete approximation to $\tilde{p}(\bar{x} ; \xi, \Delta)$.

By this procedure $\tilde{p}(\bar{x} ; \xi, \Delta)$ should be real and aliasing frequencies should have a small amplitude.

\section{References}

Baschek, B., Efimov, G. V., von Waldenfels, W., \& Wehrse, R. 1997a, A\&A, 317, 630

Baschek, B., Grüber, C., von Waldenfels, W., \& Wehrse, R. 1997b, A\&A, 320, 920

Gradshteyn, I. S., \& Ryzhik, I. M. 1980, Table of Integrals, Series, and Products (Academic Press, New York)

Feller, W. 1966, An Introduction to Probability Theory and Its Applications, vol. II (John Wiley \& Sons, New York)

Fisz, M. 1978, Wahrscheinlichkeitsrechnung und mathematische Statistik (VEB Deutscher Verlag der Wissenschaften, Berlin)

Labs, D. 1951, Zs. Astrophysik, 29, 199

Mihalas, D. 1978, Stellar Atmospheres (Freeman, San Francisco)

Peraiah, A. 1984, Discrete space theory of radiative transfer, in ed. W. Kalkofen, Methods in radiative transfer (Cambridge University Press, Cambridge), 281

Reichl, L. E. 1980, A Modern Course in Statistical Physics (University of Texas Press, Austin)

Strom, S. E., \& Kurucz, R. L. 1966, JQSRT, 6, 591

Stuart, A., \& Ord, J. K. 1987, Kendall's Advanced Theory of Statistics, vol. 1, Distribution Theory (Charles Griffin, London)

Wehrse, R., \& Baschek, B. 1999, Phys. Repo., 311, 187

Wehrse, R., Baschek, B., \& von Waldenfels, W. 2000, A\&A, 359,780

Wehrse, R., von Waldenfels, W., \& Baschek, B. 1998, JQSRT, 60,963 